\title{
Simultaneous Stokes Imaging and Doppler tomography of MCVs
}

\author{
Stephen Potter, Encarni Romero-Colmenero
}

South African Astronomical Observatory, PO Box 9, Observatory 7935 , Cape Town, South Africa.

David Buckley

SALT/South African Astronomical Observatory, PO Box 9, Observatory 7935, Cape Town, South Africa.

\section{Derren Wood}

Dept. of Mechanical Engineering, University of Pretoria, Pretoria, 0002, South Africa.

\begin{abstract}
.
Stokes imaging uses polarimetric observations in order to image indirectly the accretion region on the surface of the white dwarf in magnetic cataclysmic variables (MCVs). Doppler tomography uses spectroscopic observations in order to gain insights into the velocity dynamics of the secondary star, and the ballistic and magnetically confined parts of the accretion stream. Until now, both of these techniques have been applied separately. We present and compare the results of applying both techniques to simultaneous spectroscopic and polarimetric observations of MCVs.
\end{abstract}

\section{Stokes Imaging}

Stokes imaging models photo-polarimetric observations of MCVs in order to image indirectly the cyclotron emission region on the surface of the white dwarf in terms of its shape, size and location. It is the first technique to do this in an objective manner. It uses a genetic algorithm (eg Charbonneau 1995) to "breed" randomly generated solutions for the shape, size and location of the emission region. It then finds the smoothest solution using a regularisation term. Details of this technique can be found in Potter, Hakala, \& Cropper (1998).

There are, however, several problems with this technique. As can be seen from figure 2 of Potter et al.(2001) for example, the fit to the data appears remarkably good in some places and not so good in others. The aim of Stokes imaging is to fit the general gross features of the observations, but not fit the small scale details that either cannot be distinguished from noise or go beyond the assumptions of the cyclotron model. This is mostly controlled by the choice of the $\Lambda$ parameter used in the calculation of the smoothness of the solution (see equation 2 of Potter et al. 1998). By adjusting $\Lambda$, the smoothness of the image, 

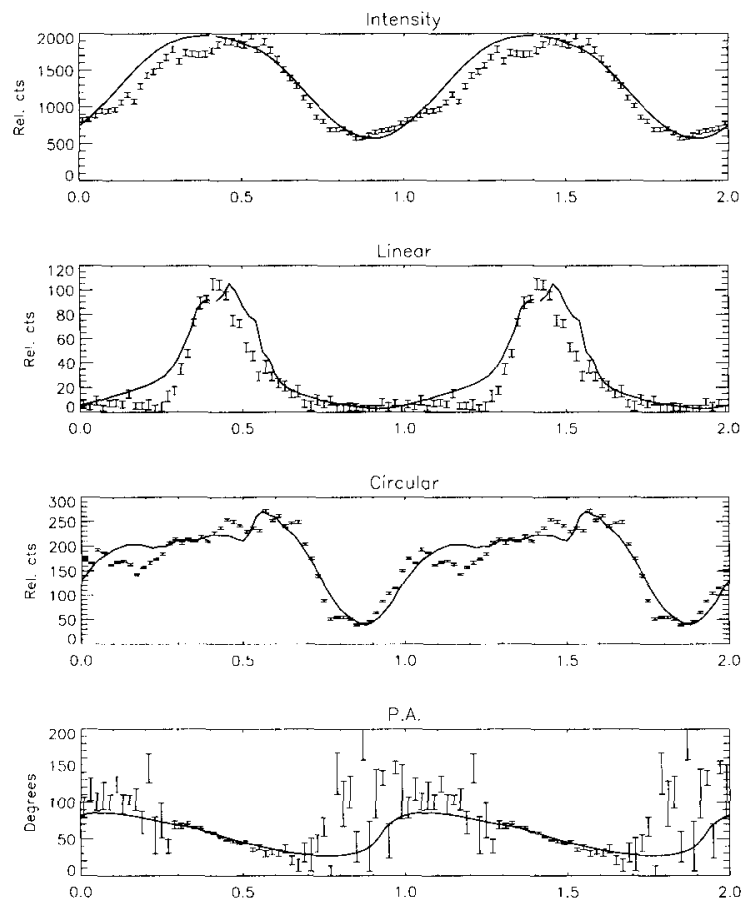

Figure 1. Optical polarimetric observations (April 2000) of the polar V834 Cen. Obtained using the University of Cape Town photopolarimeter on the $1.9 \mathrm{~m}$ of the South African Astronomical Observatory. Solid curves show the optimised model from the new Stokes imaging.

and in turn the goodness of the fit to the data, can be changed. The choice of $\Lambda$ is somewhat arbitrary and, as a result, the size of the emission region is predetermined; thus the uniqueness of the solution is uncertain. Without the regularisation term the number of possible solutions is almost infinitely large.

\subsection{New methodology}

We have removed this ambiguity by eliminating the regularisation term from the calculation of the smoothness of the solutions, leaving the $\chi^{2}$ term only. Instead, the technique is now constrained to produce and breed a single emission region of any shape, size and location per solution.

By running the technique several times, each starting with a different set of randomly generated solutions, we find that in each case the final solution is slightly different. However, a close inspection of the model solutions reveals that there are only subtle differences in the fits, as one solution may have fit one part of the light curves slightly better than another solution. All the image solutions predict a region at the same location with roughly the same shape and 


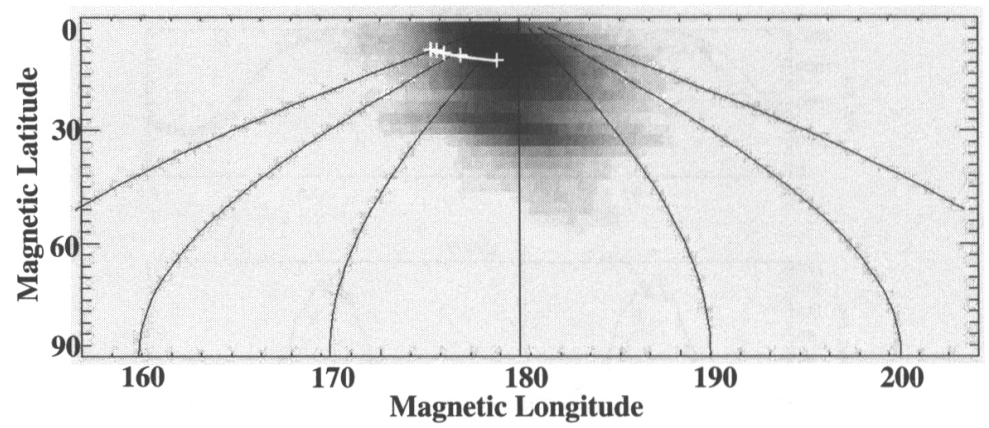

Figure 2. Probability map of the cyclotron emission region of V834 Cen from Stokes imaging. Joined crosses mark the footprints of magnetic field lines whose geometry is derived from Doppler tomography of simultaneous spectroscopic observations.

size, and there are only subtle differences in the brightness distribution from one image to the next. It therefore appears that each execution of the technique is finding a local minimum in the multidimensional parameter space, but they all are located quite close within the global minimum. Where the fits appear to be better, it is difficult to distinguish whether the technique is fitting noise or real variations in the cyclotron emission.

Therefore, instead of choosing one image as the final solution, we construct an image from the top 10 percent of 100 solutions (based on $\chi^{2}$ fit of the model to the data) by simply taking their average. This average image now represents the most probable solution for the shape, size and location of the cyclotron emission region. Similarly, the model light curves are constructed by taking the average of the top 10 percent solutions.

Figures 1 and 2 show the model fit and image solution using the new technique. This time the model light curve appears smoother (compared with figure 2 of Potter et al. 2001). This is because of two reasons. Firstly, the averaging process smears out any sharp features. Secondly, the technique does not allow for additional isolated emission regions anymore. The image is now a probability map with the brightest regions showing the most probable location of the cyclotron emission region.

\section{Doppler tomography}

The main objective of this study is to compare the results of Stokes imaging with Doppler tomography. Figure 3 shows trailed HeII 4686 spectra taken simultaneously with the polarimetry presented above. Also shown in Figure 3 is the Doppler tomogram (Spruit code 1998) and the reproduced trailed spectra.

Upon close inspection of the trailed spectra, one can see that it consists of at least three components. This is borne out in the tomogram, which shows 

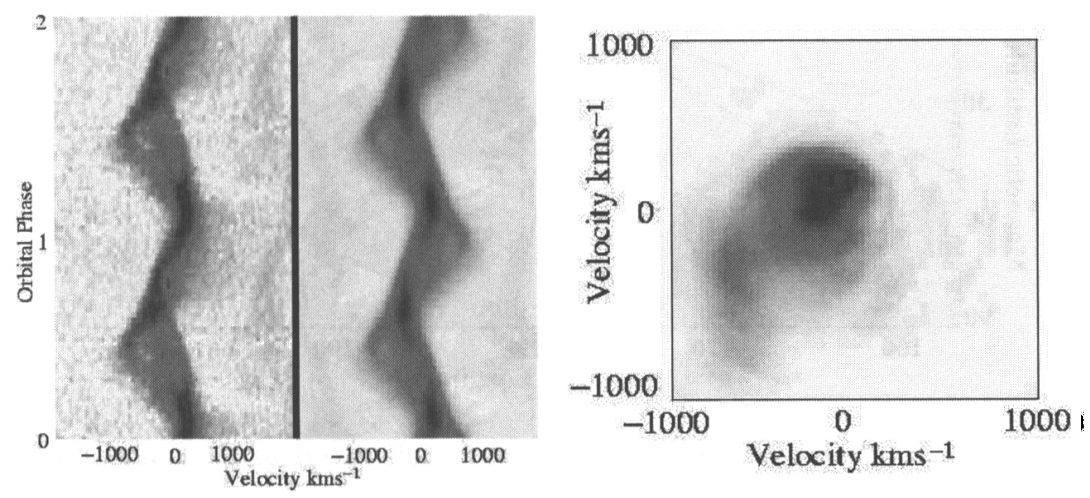

Figure 3. From left to right respectively: Trailed HeII 4686 observations (obtained during April 2000 using the Cassegrain spectrograph on the $1.9 \mathrm{~m}$ of the South African Astronomical Observatory), reproduced trailed spectra and the Doppler tomogram (Spruit code 1998)

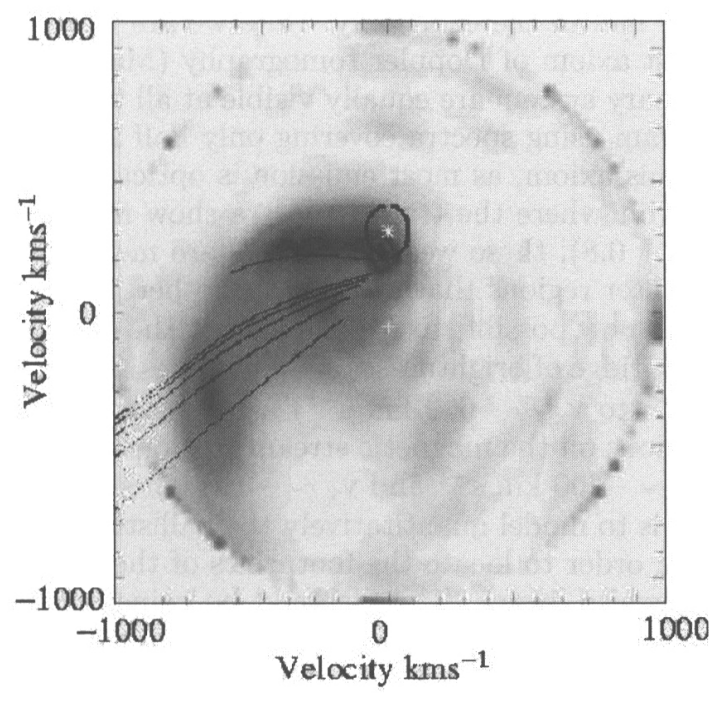

Figure 4. Doppler tomogram constructed using spectra taken between orbital phases $0.3-0.8$. Solid black curves represent the Roche lobe of the secondary and the ballistic and magnetic trajectories, calculated assuming a single particle trajectory. 


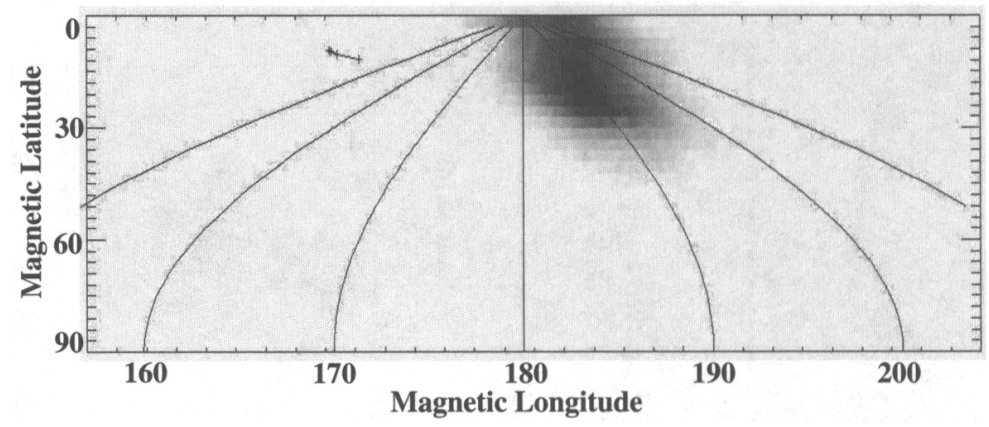

Figure 5. As for Figure 2 except the dipole offset angle (longitude) differs by 36 degrees.

emission at the location of the secondary star, the ballistic stream and perhaps part of the magnetically confined stream. However, the brightness scale of the tomogram is dominated by emission from the secondary star and around the L1 point. Consequently, the accretion stream is somewhat difficult to discern.

In Fig. 4 we show a tomogram constructed using spectra taken between phases $0.3-0.8$, ie half of the orbit only. Here we are taking advantage of the violation of the first axiom of Doppler tomography (Marsh 2001), namely that all points in the binary system are equally visible at all times, so it is possible to construct a tomogram using spectra covering only half an orbit. In our case we obviously violate this axiom, as most emission is optically thick. Therefore, by choosing half an orbit where the trailed spectra show multiple structures most clearly (phases $0.3-0.8$ ), these weaker features are more enhanced due to the absence of the brighter regions that dominate at other phases.

In Figure 4 it is now possible to see emission at the expected location of the ballistic stream (the ridge of brightness starting at $\mathrm{v}_{\mathrm{y}} \sim 20 \mathrm{~km} \mathrm{~s}^{-1}$ and extending from $\mathrm{v}_{\mathrm{x}} \sim 0 \mathrm{~km} \mathrm{~s}^{-1}$ to $\mathrm{v}_{\mathrm{x}} \sim-600 \mathrm{~km} \mathrm{~s}^{-1}$ ) and emission at locations that are consistent with regions on the magnetic stream (regions of emission centered on $\mathrm{v}_{\mathrm{y}} \sim 0 \mathrm{~km} \mathrm{~s}^{-1}, \mathrm{v}_{\mathrm{x}} \sim-200 \mathrm{~km} \mathrm{~s}^{-1}$ and $\left.\mathrm{v}_{\mathrm{y}} \sim-300 \mathrm{~km} \mathrm{~s}^{-1}, \mathrm{v}_{\mathrm{x}} \sim-700 \mathrm{~km} \mathrm{~s}^{-1}\right)$.

Our next step is to model quantitatively the ballistic and magnetic streams of the tomogram in order to locate the footprints of the magnetic field lines on the surface of the white dwarf. These should be coincident with the location of the cyclotron emission region, thus we can compare this location with the prediction for the location of the cyclotron emission region from Stokes imaging.

On the tomogram of Figure 4 we have overplotted a model trajectory computed using a single particle under gravitational and rotational influences. No additional drag terms due to the magnetic field etc. are included. The particle initially follows a ballistic path from the $\mathrm{L} 1$ point and is then constrained to follow a dipolar field line down to the surface of the white dwarf. The velocity of the particle in the direction of the field is conserved when it attaches onto a field line. 
The Doppler velocities are dependent on the inclination of the binary system, the magnetic dipole angles and the mass ratio of the binary. We used the same values as those for Stokes imaging, namely an inclination of 50 degrees and dipole offset angles (latitude and longitude) of 15 and 36 degrees respectively, consistent with those calculated by Ferrario et al. (1992) and Schwope et al. (1993). The magnetic dipole is also offset by -0.1 white dwarf radii along the dipole axis, consistent with the findings of Ferrario et al. (1992). A mass ratio $M_{1} / M_{2}=5$ and white dwarf mass of $0.7 \mathrm{M}_{\odot}$ were used, consistent with Schwope et al. (1993) and Ramsay (2000).

With this geometry, the single particle ballistic stream traces the ballistic emission fairly well. The ballistic trajectory is calculated up to a point that coincides with the end of the ballistic emission seen in the tomogram. At five equally spaced time intervals, starting from the L1 point to the end of the ballistic stream, dipole trajectories are calculated from the ballistic stream down to the surface of the white dwarf. These are represented by the five diagonal lines extending from near the origin to the bottom left corner of the tomogram. The calculated dipole trajectories overlap with the emission seen close to the origin and with the emission centered on $\mathrm{v}_{\mathrm{x}} \sim-700 \mathrm{~km} \mathrm{~s}^{-1}$ and $\mathrm{v}_{\mathrm{y}} \sim-300 \mathrm{~km} \mathrm{~s}^{-1}$. According to the calculated dipole trajectories, these two areas correspond to the coupling region and the highest point above the orbital plane where the dipole trajectory "turns over", respectively. However, it should be noted that the inclination of V834 Cen is thought to be not very high and, in addition, the out of plane velocities of the dipole trajectory will lead to a smearing effect of the corresponding emission in the Doppler tomograms.

\section{Do they agree?}

Having obtained a set of dipole trajectories, their end points on the surface of the white dwarf are calculated. This gives a quantitative idea of the approximate location, length and orientation of the shocked region that forms at the base of the accretion stream. In order to gain deeper insights into the actual density structure and emission profile of the shock, a much more detailed model than the single particle trajectory is required.

However, our simple model can still be compared for consistency with the predictions from Stokes imaging. Overplotted in Figure 2 are the end points of the five dipole trajectories. As can be seen from the figure, the dipole footprints lie quite close to the region of highest probability predicted by Stokes imaging. Furthermore, the orientation and extent of the Stokes image (at least the highest probability part) is also in agreement with the dipole footprints.

\section{Summary, Discussion and Future Work}

We have compared the results from Stokes imaging with Doppler tomography. Good agreement was found in terms of the location, orientation and extent of the emission region from Stokes imaging with the footprints of the magnetically confined accretion flow calculated from modelling the Doppler tomogram.

It is indeed encouraging, that the two techniques are giving compatible results. Currently this study benefits from the fact that V834 Cen is a relatively 
well studied MCV and, consequently, the system parameters (inclination, dipole offset angle etc.) have been calculated to a good degree of confidence. Hence the result should agree. Even so, the two techniques do not overlap exactly (Figure 2). Undoubtedly, there is a set of system parameters in which the two techniques would overlap exactly.

For other less studied MCVs, where the system parameters are not so well known, Stokes imaging and Doppler tomography can, obviously, place some constraints on the system parameters independently (eg. see Potter, Cropper, \& Hakala 2000).

However, in Figure 5 we show the results of applying Stokes imaging and Doppler tomography to the same observations as above, but with the dipole offset angle (longitude) offset by 36 degrees from the previous value. Independently, the two techniques still give good results. However, when the two results are compared, there is not a very good agreement. Therefore, by comparing the results of the two techniques, better constraints of the system parameters can be achieved.

A more detailed description of the new Stokes imaging technique and a detailed investigation into the combination of Stokes imaging and Doppler tomography will be presented elsewhere.

\section{References}

Charbonneau, P., 1995, ApJS, 101, 309

Ferrario, L., Wickramasinghe, D. T., Bailey, J., Hough, J. H., \& Tuohy, I. R., 1992, MNRAS, 256, 252

Marsh, T., 2001, in LNP Conf. Proc., 573, "Astrotomography", eds. H.H.J. Boffin, D. Steeghs \& J. Cuypers (Heidelberg: LNP), 1

Potter, S.B., Hakala, P.J., \& Cropper, M. 1998, MNRAS, 297, 1261

Potter, S.B., Cropper, M., \& Hakala, P.J., 2000, MNRAS, MNRAS, 315, 423

Potter, S.B., Romero-Colmenero, E., Buckley, D.A.H., Cropper, M., Hakala, P.J., 2001, in LNP Conf. Proc., 573, "Astrotomography", eds. H.H.J. Boffin, D. Steeghs \& J. Cuypers (Heidelberg: LNP), 244

Ramsay, G.T.B., 2000, MNRAS, 314, 403

Spruit, H.C., 1998, astro-ph/9806141

Schwope, A.D., Thomas, H.-C., Beuermann, K., \& Reinsch, K., 1993, A\&A, 267,103 